\title{
Variation in Pathogenicity and Aggressiveness of Erysiphe necator from Different Vitis spp. and Geographic Origins in the Eastern United States
}

\author{
Omer Frenkel, Marin Talbot Brewer, and Michael G. Milgroom
}

Department of Plant Pathology and Plant-Microbe Biology, Cornell University, Ithaca, NY 14853.

Accepted for publication 17 June 2010.

\begin{abstract}
Frenkel, O., Brewer, M. T., and Milgroom, M. G. 2010. Variation in pathogenicity and aggressiveness of Erysiphe necator from different Vitis spp. and geographic origins in the eastern United States. Phytopathology 100:1185-1193.

Eastern North America is considered the center of diversity for many Vitis spp. and for the grape powdery mildew pathogen, Erysiphe necator. However, little is known about populations of E. necator from wild Vitis spp. We determined the phenotypic variation in pathogenicity and aggressiveness of E. necator among isolates from wild and domesticated Vitis spp. from diverse geographic regions in the eastern United States. To test pathogenicity, we inoculated 38 E. necator isolates on three wild Vitis

which complete host specialization was evident; it was only susceptible to isolates collected from $V$. rotundifolia. All isolates, regardless of source host, were pathogenic on the other Vitis spp. We found no differences in components of aggressiveness latent period and lesion size among isolates from different source hosts when inoculated on V. vinifera, which is highly susceptible to powdery mildew. However significant variation was evident among isolates on the more resistant $V$. labruscana 'Niagara'. Isolates from the wild species $V$. aestivalis were the most aggressive, whereas isolates from $V$. vinifera were not more aggressive than isolates from other source hosts. Greater aggressiveness was also detected among isolates from the southeastern United States compared with isolates from the northeastern United States.
\end{abstract} spp., two commercially grown hybrids and the European wine grape, Vitis vinifera. V. rotundifolia (muscadine grape) was the only host species on
Additional keywords: durability, Uncinula.
Adaptation and fitness of individual pathogen genotypes is partially determined by two distinct factors: pathogenicity (i.e., the ability to infect particular host species or genotypes) and aggressiveness (i.e., the quantitative component of pathogenicity). In previous studies, large variation in pathogenicity and aggressiveness has been detected within and between pathogen populations. Such variation could arise due to the agroecological environment, host diversity, cultural practices, and sexual recombination.

The grape powdery mildew fungus, Erysiphe necator (syn. Uncinula necator), is an obligate biotroph on host species in the family Vitaceae, particularly in the genus Vitis but also in Parthenocissus and Ampelopsis $(2,20)$. Although powdery mildew is one of the most economically important diseases of grape worldwide (39), many commercially grown grape cultivars are highly susceptible to E. necator because they are derived from the European wine grape, Vitis vinifera, which was not exposed to this pathogen during its evolution (38). Eastern North America is the center of diversity for many wild species of Vitis, with relatively high levels of resistance to some of the most notorious diseases and pests of grapevines, including powdery mildew. Native grapevine species such as V. labrusca, V. rupestris (12), $V$. aestivalis (24), and $V$. rotundifolia (syn. Muscadinia rotundifolia) (35), which probably coevolved with E. necator in North America, possess varying levels of resistance to powdery mildew. Resistance to powdery mildew in North American Vitis spp. is determined by diverse mechanisms. For example, resistance in $V$.

Corresponding author: M. G. Milgroom; E-mail address: mgm5@ cornell.edu

* The $\boldsymbol{e}$-Xtra logo stands for "electronic extra" and indicates that the online version contains a supplemental table.

doi:10.1094/PHYTO-01-10-0023

(c) 2010 The American Phytopathological Society rotundifolia is associated with Run1, a dominant, complex locus that contains two families of resistance gene analogs. Resistance in plants with Runl is mediated through a hypersensitive response (11). Alternatively, resistance may be associated with constitutive expression of pathogenesis-related (PR) genes, such as genes encoding $\beta$-1,3-glucanases (PR-2) and chitinases (PR-3), in addition to other quantitative resistance mechanisms found in North America Vitis spp. $(18,24)$.

Few studies have been conducted to study variation in aggressiveness in E. necator. In Europe, these efforts have focused on comparisons between genetic groups A and B (sampled only from $V$. vinifera), which represent distinct lineages of $E$. necator derived from populations in North America (3). Some studies found significant differences between groups A and B in infection efficiency, sporulation, latent period, and colony expansion rates $(33,41)$. In contrast, much less effort has been devoted to studying variation in aggressiveness in North America. In a comprehensive study of host specialization, Gadoury and Pearson (20) tested 35 E. necator isolates from different Vitis spp. on two experimental hosts: V. vinifera and labrusca hybrid cv. Katawba. They found no differences among isolates in latent period or colony expansion rates on $V$. vinifera. In contrast, they did find significant differences in colony expansion rates when they inoculated the more resistant 'Katawba' with the same isolates. However, their sampling of isolates was mostly done in the northeastern United States (New York and New England), with a few isolates from the southeastern United States. In addition, they focused mostly on isolates sampled from $V$. vinifera and hybrids of $V$. vinifera with North American species from commercial vineyards. As a consequence, no information, to our knowledge, is available on the aggressiveness of populations from wild Vitis spp. such as $V$. aestivalis or from cultivated (or wild) muscadine grape (V. rotundifolia). These latter two species are potentially important 
hosts of E. necator because $V$. rotundifolia (36) and cultivars of predominantly $V$. aestivalis ancestry (e.g., cv. Norton) (44) are grown commercially in the southeastern and central United States, sometimes sympatrically with $V$. vinifera.

Although populations of E. necator in Europe and Australia are composed of two genetically distinct lineages (groups A and B) $(10,42)$, the structure of North American populations is markedly different. Brewer and Milgroom (3) recently reported that populations of E. necator in the eastern United States are genetically diverse, as expected in a native population, and are not structured by distinct lineages. Our overall hypothesis is that, because the populations of $E$. necator in the eastern United States are diverse with respect to genotypes, wild Vitis host species (and powdery mildew resistance mechanisms among them), geography, and climate, we expect to find large variation in pathogenicity and aggressiveness on different host species. Our specific aims were to quantify the variation in E. necator populations from different Vitis hosts and regions in the eastern United States with respect to their pathogenicity and aggressiveness.

\section{MATERIALS AND METHODS}

Collection and maintenance of fungal isolates. We collected E. necator in 2007 to 2009 and concentrated our search on the more common host species in the eastern United States (we refer to these as "source hosts") (Table 1). We collected E. necator from (i) V. riparia, which is mostly found in the northeastern United States; (ii) V. aestivalis, which can be found across the eastern United States but is most common at high elevation in the southeast; (iii) V. labrusca, which is more common near coastal areas of New England and in the mid-Atlantic states, but can be also found at high elevations in the southeast; (iv) $V$. rotundifolia (muscadine grape), which is grown commercially and grows wild in the southeastern United States, from the coastal areas to the lower slopes of the Appalachian mountains; and (v) V. vinifera, the European wine grape, which is grown commercially across the eastern United States (21). Because powdery mildew cannot be easily detected on wild species, we sampled wherever we or our colleagues encountered it in various locations in the following northeastern states: Massachusetts (MA), New Hampshire (NH), Connecticut (CT), New York (NY), New Jersey (NJ), Ohio (OH), and Michigan (MI). In the southeast, we concentrated our sampling in two distinct areas. First, we sampled at low elevations in North Carolina (NC), South Carolina (SC), and Georgia (GA), where powdery mildew could only be found on $V$. vinifera and $V$. rotundifolia. Second, we sampled at higher elevations in the Appalachian Mountains in GA, NC, and Virginia (VA) from all five Vitis spp. In addition, we sampled isolates randomly from commercial vineyards of $V$. vinifera in $\mathrm{NC}$ and GA, where mildew was more common. Approximate collection sites are presented in Figure 1.

TABLE 1. Sampling of Erysiphe necator from diverse hosts in the northeastern (New York, Pennsylvania, Ohio, Michigan, Massachusetts, and New Hampshire) and southeastern (Georgia and North Carolina) United States

\begin{tabular}{lccc}
\hline Source host & $\begin{array}{c}\text { No. of } \\
\text { isolates }\end{array}$ & $\begin{array}{c}\text { Northeastern } \\
\text { United States }\end{array}$ & $\begin{array}{c}\text { Southeastern } \\
\text { United States }\end{array}$ \\
\hline Vitis riparia & $13^{\mathrm{a}}$ & 11 & 1 \\
V. aestivalis & $16^{\mathrm{a}}$ & 3 & 11 \\
V. labrusca & 8 & 4 & 4 \\
V. rotundifolia & 10 & 0 & 10 \\
V. vinifera & 13 & 6 & 7 \\
Total & 60 & 24 & 33 \\
\hline
\end{tabular}

a One isolate from $V$. riparia was collected in Kansas and two isolates from $V$. aestivalis were collected in Virginia and Kansas; these isolates were not included in either the northeastern or southeastern United States samples.

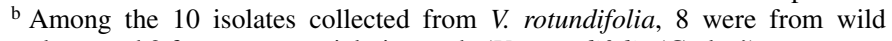
plants and 2 from commercial vineyards ( $V$. rotundifolia 'Carlos').
Isolates were maintained as described by Evans et al. (16). Briefly, V. vinifera 'Cabernet Sauvignon' was grown in the greenhouse to produce leaves for culturing E. necator. The fourth youngest leaves were collected and surface sterilized in $0.6 \%$ sodium hypochlorite for $2 \mathrm{~min}$, rinsed twice in sterile distilled water, and dried in a sterile laminar flow hood. Leaves were placed in petri dishes containing $20 \mathrm{ml}$ of $2 \%$ water agar to maintain sufficient moisture. Mass transfers of conidia and mycelium from colonies on infected leaves collected in the field were made to surface-sterilized leaves under a dissecting microscope $(\times 200)$ using a sterile pipette tip. Another transfer was made from the resulting colony at least once to avoid contamination. After at least two transfers, an individual chain of conidia was transferred from each mildew colony, with a sterile pipette or with an eyelash fastened to a Pasteur pipette, to a new leaf, as described by Gadoury and Pearson (20). Resulting single-conidial-chain isolates were maintained on leaves in a growth room at 20 to $25^{\circ} \mathrm{C}$ in 12 -h cycles of light and darkness. Colonies were transferred to new leaves every 18 to 24 days.

Host specialization on Vitis spp. and hybrids. We determined the ability of E. necator isolates from different Vitis hosts to colonize leaf tissue (i.e., pathogenicity) of wild and domesticated Vitis spp. on the following six hosts ("inoculated hosts"): (i) V. vinifera Cabernet Sauvignon, (ii) V. rotundifolia var. munsoniana

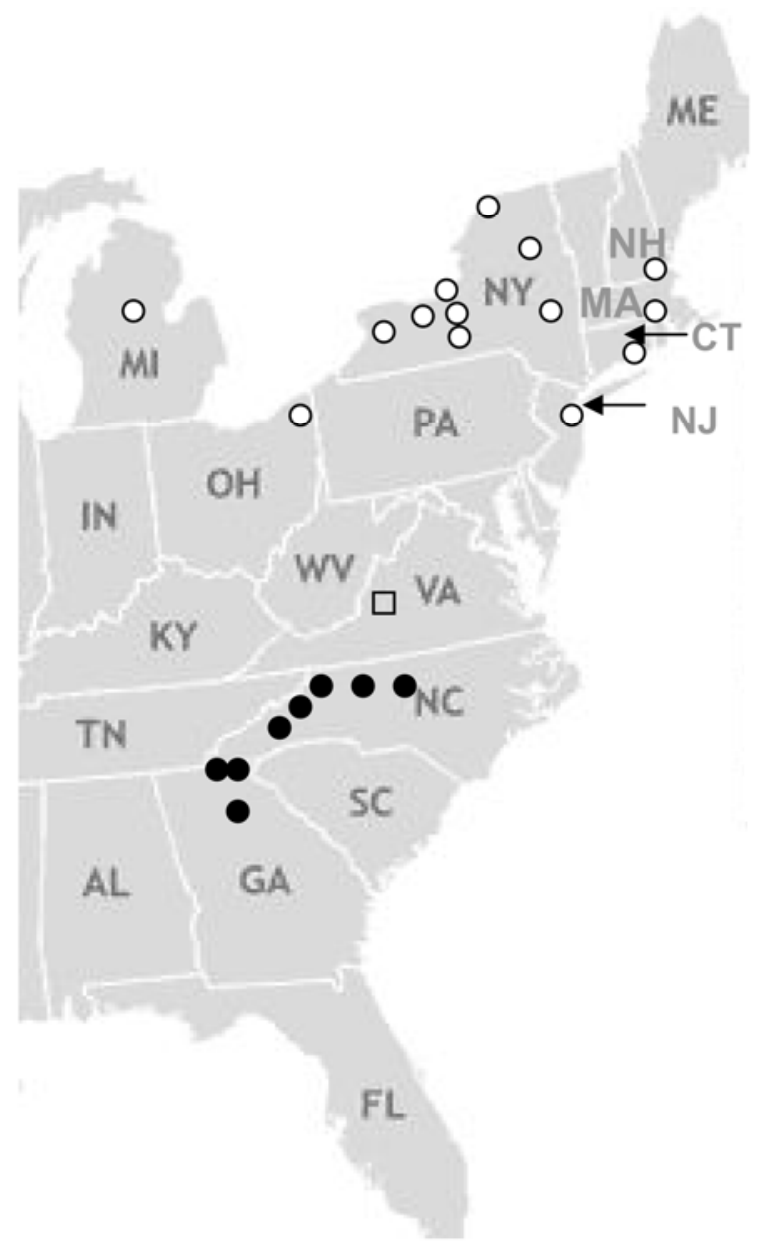

Fig. 1. Approximate locations where we sampled Erysiphe necator from wild and commercially grown Vitis spp. in the eastern United States during 20072009. $\bigcirc=$ Sample from the northeastern United States $(n=24)$ : New Hampshire (NH), Massachusetts (MA), Connecticut (CT), New York (NY), New Jersey (NJ), Ohio $(\mathrm{OH})$, and Michigan (MI). $=$ Samples from the southeastern United States $(n=33)$ : North Carolina (NC) and Georgia (GA). $\square=$ Samples from states which were excluded from the northeast versus southeast analysis (see Materials and Methods): Virginia (VA) and Kansas (Lawrence, KS, not shown on map). 
(accession DVIT 2735; United States Department of AgricultureAgricultural Research Service [USDA-ARS] National Clonal Germplasm Repository, Davis, CA), (iii) V. aestivalis (accession REM 59-77; USDA-ARS Plant Genetic Resources Unit, Geneva, NY), and iv) V. riparia growing wild in Ithaca, NY. In addition, we used two hybrids: (v) cv. Niagara, which is a $V$. vinifera $\times V$. labrusca hybrid (Double A vineyards, Fredonia, NY) and (vi) cv. Norton (syn. 'Cynthiana'), which is a cultivar of predominantly $V$. aestivalis ancestry, probably derived by hybridization with $V$. vinifera (44). Both hybrids are commercially grown in the eastern United States and considered less susceptible to E. necator than $V$. vinifera. All Vitis spp. were grown from cuttings and received a cold treatment before being potted in 4-liter pots and grown at $25^{\circ} \mathrm{C}$ in the greenhouse. The only exception was $\mathrm{V}$. riparia, whose leaves were collected from a single wild vine in May 2009. Fourth-youngest leaves from each of the six inoculated hosts were surface sterilized and placed in petri dishes as described above. We inoculated five to nine isolates collected from each of the five source hosts (total $n=38$ ). Each inoculation was conducted six times with one inoculation per leaf. Inoculation was conducted by applying $\approx 200$ conidia to the center of the leaf with a sterile pipette tip. Plates were wrapped with Parafilm and placed in growth chambers at $22^{\circ} \mathrm{C}$ under a regime of 12-h cycles of darkness and light. Leaves were inspected under a dissecting microscope $(\times 200)$ to detect colony growth and sporulation beginning 5 days after inoculation and every 3 to 4 days thereafter until the maximum of 28 days post inoculation.

Aggressiveness components: latent period and lesion size. Aggressiveness was estimated on fourth-youngest leaves of $V$. vinifera cv. Cabernet Sauvignon and Niagara. 'Cabernet Sauvignon' is considered highly susceptible to powdery mildew, whereas labrusca hybrid cultivars are less susceptible (39). Gadoury and Pearson (20) demonstrated significant variation in aggressiveness of E. necator on labrusca hybrid cv. Katawba but not on $V$. vinifera. Therefore, we hypothesized that cv. Niagara, with its strong background from $V$. labrusca, might be comparable for showing differences in aggressiveness. We also attempted to use $V$. aestivalis as a host for estimating aggressiveness but, because many of the detached leaves of this species decomposed after $<10$ days in our experimental conditions, we excluded it from these experiments. Sixty isolates from the five source hosts were used in this study (Table 1). Aqueous suspensions of conidia were prepared by vortexing excised mildew colonies 8 to 10 days after inoculation in $1.5-\mathrm{ml}$ microcentrifuge tubes with $1 \mathrm{ml}$ of water and $0.05 \%$ Tween 20 . After counting with a hemacytometer, the conidial suspension was adjusted to $1 \times 10^{5}$ conidia/ml and each leaf was inoculated with a $10-\mu l$ droplet. Inoculated leaves were left in the laminar flow hood for 30 to $40 \mathrm{~min}$ until the droplet fully evaporated. Petri dishes were wrapped with Parafilm and placed in completely randomized locations in a growth chamber at $22^{\circ} \mathrm{C}$ under a 12 -h regime of light and darkness. We were concerned that disease would develop too quickly under optimal conditions and, as a result, differences would not be distinguishable between isolates. Therefore, experiments were conducted at $22^{\circ} \mathrm{C}$ rather than the optimal temperature of $25^{\circ} \mathrm{C}$ to slow pathogen growth (9). Latent period (i.e., the time from inoculation until the appearance of the first conidia) was determined by daily inspection for conidia under a dissecting microscope $(\times 400)$. Inspection took place each morning from day 4 to day 10 . Lesion area was calculated from colony diameters measured in two perpendicular directions ( $a$ and $b$ ) 13 days after inoculation. Because the two diameters were often unequal, we calculated lesion area as an ellipse ( $\pi a b / 4$, in square millimeters). Each treatment (inoculated host species-isolate) had eight replicates and the experiment was conducted twice.

Statistical analyses were performed with the JMP 5.0 software for Windows (SAS Institute, Cary, NC). To conduct an analysis of variance on non-normally distributed data and unequal variances, a rank transformation was applied to all results (6). We used the data from the 60 isolates to assess differences in aggressiveness based on source hosts by using a mixed model with the source host and inoculated host (V. vinifera or 'Niagara') as fixed effects, while isolates were treated as random effects. The experiment was considered as an additional fixed effect.

To test whether aggressiveness differed between geographic regions, we divided isolates into those from the northeastern United States (NY, MA, NH, CT, NJ, OH, and MI; $n=24$ ) and those from southeastern United States (GA and NC; $n=33$ ). Three isolates collected in VA or Kansas were excluded from the analysis because they were not geographically and climatically aligned with either region. For this test, we used a second mixed model in which geographic region, inoculated host, and experiment were fixed effects and isolates were random effects. Multiple comparisons of the means were conducted with the Tukey-Kramer honestly significant differences (HSD) test $(\alpha=0.05)$.

\section{RESULTS}

We isolated E. necator from four wild species during the growing seasons of 2007-09 (Table 1; Fig. 1). Symptoms and signs of powdery mildew on $V$. aestivalis, $V$. riparia, and $V$. labrusca were very similar to those on $V$. vinifera and commercial hybrids. In the southeastern United States, source hosts were found from sea level at Myrtle Beach, SC up to $1,250 \mathrm{~m}$ in the Blue Ridge Parkway (Buncombe and Haywood Counties, NC). However, E. necator was only found on V. riparia, V. aestivalis, and $V$. labrusca at elevations of 875 to $1,180 \mathrm{~m}$, and mostly in shaded areas. Interestingly, we frequently found severely diseased plants adjacent to plants of the same species with no symptoms of powdery mildew. Powdery mildew was found on $V$. rotundifolia growing wild on the lower slopes of the Appalachians (Panther Creek Falls, Clarksville, GA, elevation 450 m; and De Soto Falls, Lumpkin County GA, elevation $635 \mathrm{~m}$ ) and from commercial vineyards in central NC (Hurdle Mills, elevation $187 \mathrm{~m}$ ) and GA (Ringgold, elevation $237 \mathrm{~m}$ ). Powdery mildew symptoms on $V$. rotundifolia differed from those on other Vitis spp. Colonies appeared mostly on the abaxial side of the leaves and lesions were generally smaller, with dense hyphal growth. In the northeastern United States, we found powdery mildew on plants at elevations as low as $15 \mathrm{~m}$ (V. labrusca, Portsmouth, $\mathrm{NH})$ and up to $550 \mathrm{~m} \mathrm{(V}$. riparia, Sugar Hill State Forest, Schuyler County, NY). In the northeast, disease was less confined to shady areas than in the southeast, although no quantitative data were recorded. Cleistothecia were found in mildew colonies in most locations on all of the host species except for $V$. rotundifolia.

Host specialization on Vitis spp. and hybrids. All isolates, regardless of source host, formed mildew colonies and produced abundant sporulation on five of the inoculated hosts: $V$. vinifera, $V$. riparia, and $V$. aestivalis and the hybrids $V$. labruscana Niagara and cv. Norton (Table 2; Fig. 2). In contrast, only isolates collected from $V$. rotundifolia $(n=10)$ were pathogenic on $V$. rotundifolia. These isolates formed macroscopic colonies with abundant sporulation on $V$. rotundifolia, as well as on the other five inoculated hosts (Fig. 2). Isolates from other Vitis spp. ( $n=$ 28) produced only a few sparse hyphae, with no conidia or conidiophores, on $V$. rotundifolia. We observed necrotic epidermal cells below these hyphae, which we interpret as hypersensitive responses at the sites of attempted penetration (Fig. 2).

Variation in aggressiveness. We detected variation in latent period and lesion size among 60 isolates on both $V$. vinifera and 'Niagara'. On $V$. vinifera, the average latent period among all 60 isolates was 6.0 days, ranging between 5.1 and 8.0 days (Fig. 3A). The average lesion size was $103.2 \mathrm{~mm}^{2}$, ranging between 67.0 and $150.0 \mathrm{~mm}^{2}$ (Fig. 3C). On 'Niagara', the average latent period among all 60 isolates was 6.5 days, ranging between 5.0 and 
8.6 days (Fig. 3B). The average lesion size was $97.9 \mathrm{~mm}^{2}$, ranging between 60.3 and $146.4 \mathrm{~mm}^{2}$ (Fig. 3D).

Effect of original source hosts on aggressiveness. Linear models were used to compare rank transformations of latent period and lesion size among isolates from five source hosts (Table 3). Analysis of variance (ANOVA) models were significant $(P<0.001)$ and explained 29.6 and $22.8 \%$ of the observed variation of latent period and lesion size, respectively. The correlation between the means for latent period and lesion size was significant $(P=0.01)$ but weak $(r=0.33)$.

For both latent period and lesion size, the source host $\times$ inoculated host interactions were significant $(P=0.003$ and 0.002 , respectively) (Table 3). Therefore, we analyzed the effect of source host on $V$. vinifera and 'Niagara' separately. The variance component of the random effect (isolates) on latent period was $40.5 \%$. The experiment effect was not significant for latent period; therefore, we combined results of the two experiments. On $V$. vinifera, mean latent period varied from 5.9 to 6.2 days but no significant differences were detected among source hosts (Fig. 4A). In contrast, we found one significant difference in latent period between source hosts on 'Niagara'. Isolates from $V$. aestivalis had the shortest mean latent periods (6.1 days), which was significantly shorter than the mean latent period of isolates from $V$. riparia (6.7 days) (Fig. 4B). No significant differences were detected among means of isolates from $V$. labrusca, $V$. vinifera, and $V$. rotundifolia and any other source host (Fig. 4B).

For lesion size, the variance component of the random effect (isolates) was $15.5 \%$ (Table 3 ). Although the means for lesion size in the experiments were different, the relative differences among isolates from different source hosts were similar between experiments; therefore, we show the combined results of the experiments. Mean lesion size on $V$. vinifera varied from 97.5 to $107.8 \mathrm{~mm}^{2}$ but no significant differences were detected among isolates from different source hosts (Fig. 4C). On 'Niagara', however, lesion sizes for isolates from $V$. aestivalis $\left(108.9 \mathrm{~mm}^{2}\right)$ and $V$. riparia $\left(87.0 \mathrm{~mm}^{2}\right)$ were significantly different. Isolates from $V$. aestivalis also had significantly larger lesions than those of isolates from $V$. vinifera and $V$. rotundifolia (Fig. 4D). Mean lesion size of isolates from $V$. rotundifolia was also significantly larger than the mean lesion size of isolates from $V$. riparia. No

TABLE 2. Pathogenicity of Erysiphe necator isolated from five Vitis source hosts inoculated on four Vitis spp. and two hybrids ('Niagara' and 'Norton')a

\begin{tabular}{|c|c|c|c|c|c|c|}
\hline \multirow[b]{2}{*}{ Source hosts (origin of isolates) ${ }^{b}$} & \multicolumn{6}{|c|}{ Inoculated hosts } \\
\hline & V. vinifera & V. riparia & V. aestivalis & 'Niagara' & 'Norton' & V. rotundifolia \\
\hline$V$. vinifera $(n=8)$ & + & + & + & + & + & - \\
\hline$V$. riparia $(n=8)$ & + & + & + & + & + & - \\
\hline$V$. aestivalis $(n=8)$ & + & + & + & + & + & - \\
\hline V. labrusca $(n=5)$ & + & + & + & + & + & - \\
\hline V. rotundifolia $(n=9)$ & + & + & + & + & + & + \\
\hline
\end{tabular}

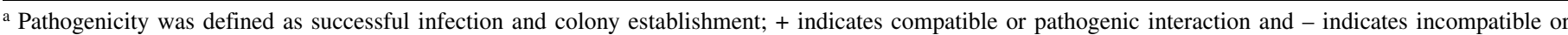
nonpathogenic interaction.

${ }^{\mathrm{b}}$ Number in parenthesis indicates the number of isolates from each source host that were used in the experiment.

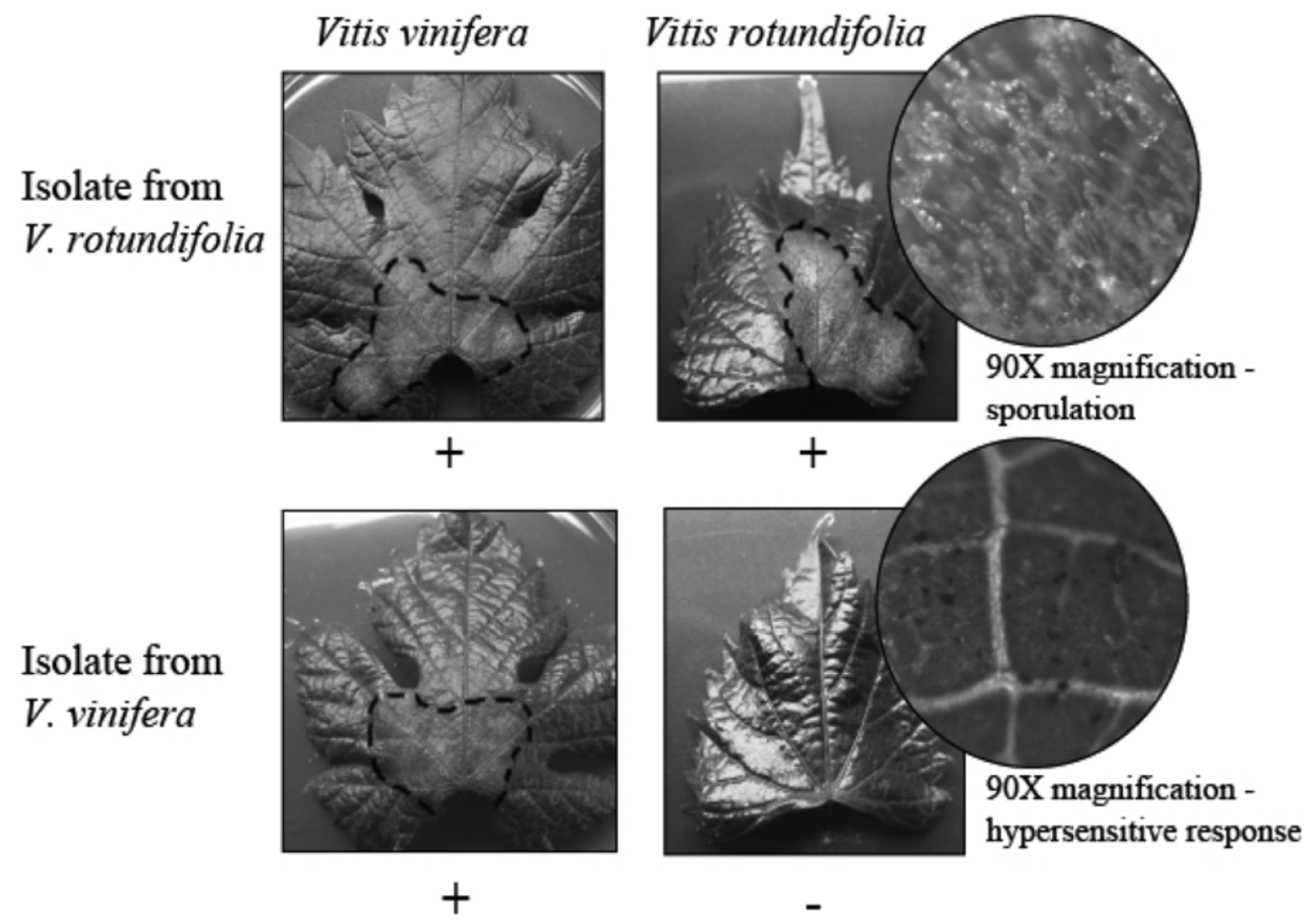

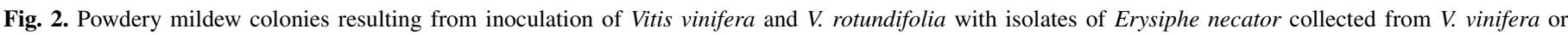

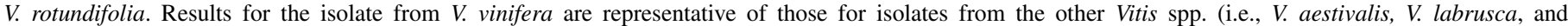

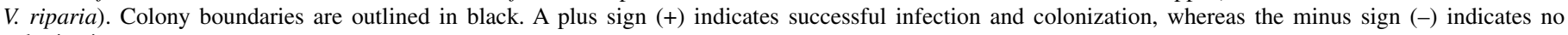
colonization. 
significant differences were detected among isolates from $V$. rotundifolia, $V$. vinifera, and V. labrusca (Fig. 4D).

The ANOVA models comparing aggressiveness of isolates from two different geographic regions, northeastern United States $(n=$ $24)$ and southeastern United States $(n=33)$, are presented in Table 4. ANOVA models were significant for latent period and lesion size and explained 29.2 and $22.8 \%$ of the observed variation, respectively. For latent period, only the main factor (inoculated host; $V$. vinifera versus 'Niagara') was significant (Table 4). The differences between the experiments were not significant and the results of the two experiments were analyzed together. No significant differences were detected for latent period between isolates from the northeastern and southeastern regions on either of the inoculated hosts (6.0 and 6.3 days, respectively, on $V$. vinifera; and 6.3 and 6.8 days, respectively, on 'Niagara') (Fig. 5A and B). For lesion size, the main factors, geographic region and inoculated host, were significant $(P=$ 0.027 and 0.002 , respectively) but not their interaction $(P=$ 0.977). The variance component of the random effect (isolates) was $14.7 \%$. The isolates from the southeast had a significantly larger lesion size than isolates from the northeast (103.9 and $95.1 \mathrm{~mm}^{2}$, respectively), regardless of which host was inoculated (Fig. 5B).
Sampling from source host was partially confounded with geographic region because isolates of $V$. rotundifolia were only found in the southeast and all but one isolate from V. riparia were found in the northeast. Therefore, testing the significance of the geographic region $\times$ source host interaction would not be meaningful. However, for the three source hosts ( $V$. aestivalis, $V$. vinifera, and $V$. labrusca) for which we had reasonable sample sizes from both regions (Table 1), we performed $t$ tests. Southeastern isolates of $V$. aestivalis had a significantly shorter mean latent period than northeastern isolates from $V$. aestivalis, regardless of which host was inoculated (5.9 versus 7.3 days on $V$. vinifera and 5.8 versus 7.1 days on 'Niagara'; $P<0.001$ ) and significantly larger mean lesion size than northeastern isolates (106.9 versus $81.2 \mathrm{~mm}^{2}$ on $V$. vinifera and 116.5 versus $78.9 \mathrm{~mm}^{2}$ on 'Niagara'; $P<0.001)$. No significant differences were found between regions when comparing isolates from $V$. vinifera $(P>$ $0.09)$ or V. labrusca $(P>0.15)$.

\section{DISCUSSION}

Centers of diversity of native hosts often correspond with the centers of diversity of their pathogens. In many cases, these centers of diversity are studied to characterize important phenotypic
Inoculated host: $V$. vinifera
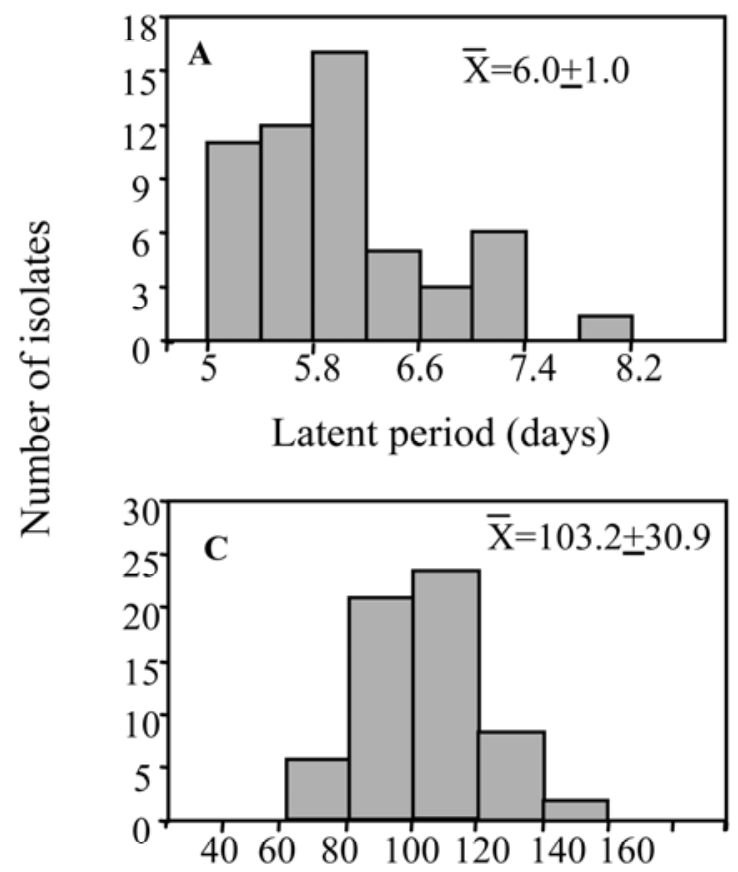

Inoculated host: cv. Niagara
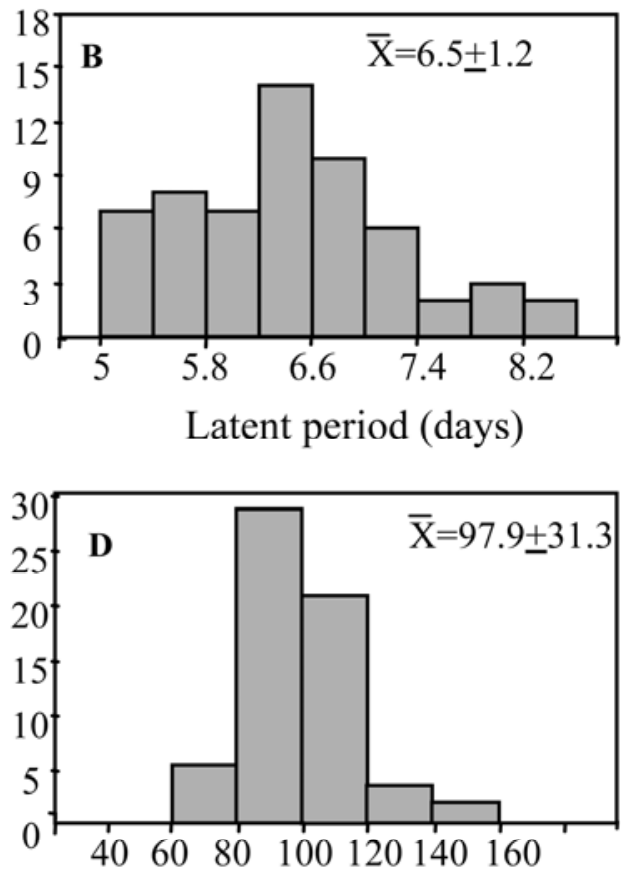

Lesion size $\left(\mathrm{mm}^{2}\right)$

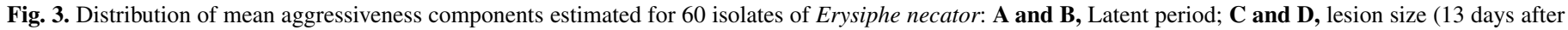

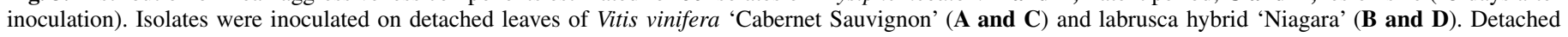
leaves were maintained at $22^{\circ} \mathrm{C}$ under a 12 -h regime of light and darkness. Mean values \pm standard deviations over all 60 isolates are presented.

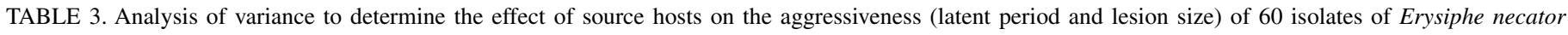
inoculated on two hosts, Vitis vinifera 'Cabernet Sauvignon' and labrusca hybrid 'Niagara'

\begin{tabular}{|c|c|c|c|c|c|c|c|}
\hline \multirow[b]{2}{*}{ Source } & \multirow[b]{2}{*}{ df } & \multicolumn{3}{|c|}{ Latent period } & \multicolumn{3}{|c|}{ Lesion size } \\
\hline & & SS & $F$ ratio & Prob $>F$ & SS & $F$ ratio & Prob $>F$ \\
\hline Source host ${ }^{\mathrm{a}}$ & 4 & 178,428 & 0.4883 & 0.7444 & 938,381 & 2.2075 & 0.0662 \\
\hline Inoculated host & 1 & $6,071,478$ & 66.4621 & $<0.0001$ & $1,841,758$ & 17.3304 & $<0.0001$ \\
\hline Source host $\times$ inoculated host & 4 & $1,447,361$ & 3.9609 & 0.0034 & $1,804,593$ & 4.2452 & 0.0020 \\
\hline Experiment & 1 & 152,137 & 1.6654 & 0.1971 & $17,223,235$ & 162.0653 & $<0.0001$ \\
\hline
\end{tabular}

a Isolates of E. necator were collected from five source hosts: V. riparia, V. aestivalis, V. labrusca, V. vinifera, and V. rotundifolia. 
traits of the host which have agricultural importance, such as disease resistance $(27,30)$. In the same manner, exploring phenotypic variation in pathogenicity and aggressiveness of the natural pathogen populations may provide significant knowledge about these traits (49), as well as inform us about host resistance mechanisms and the potential breakdown of resistance.

In this study, we challenged several of the most common wild and domesticated species in the genus Vitis in the eastern United States with a collection of $E$. necator isolates from diverse hosts and geographic origins in the eastern United States. The only combination where complete host specialization was observed was between $V$. rotundifolia and $E$. necator isolates collected from this same host species. Interestingly, all 10 isolates from $V$. rotundifolia were pathogenic on $V$. rotundifolia as well as on all other Vitis spp. we tested, whereas none of the isolates from the other Vitis spp. was pathogenic on V. rotundifolia, even though they were all pathogenic on the other Vitis hosts. V. rotundifolia (muscadine grape) differs from the other Vitis spp. in number of morphological, anatomical, and cytological characters (21). Those differences include having 40 chromosomes (whereas other Vitis spp. have 38 chromosomes) and having prominent lenticels, among other differences (21). Overall, V. rotundifolia is different enough from other Vitis spp. that it is sometimes considered as being in a different genus, Muscadinia $(21,35)$. It is possible that $V$. rotundifolia has defense mechanisms that are different from those of the other Vitis spp. tested. One example is the dominant resistance locus Runl, which is present in some $V$. rotundifolia accessions. Analysis of the Runl-mediated resistance response

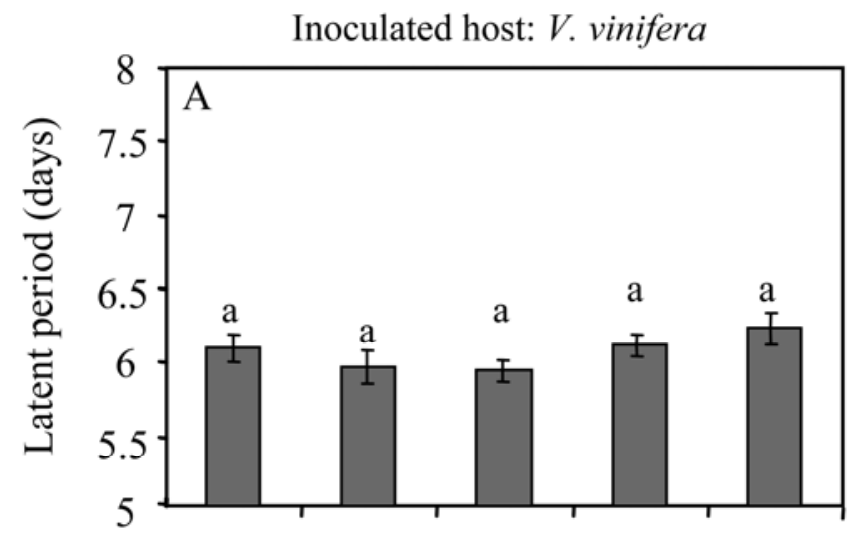

Inoculated host: cv. Niagara
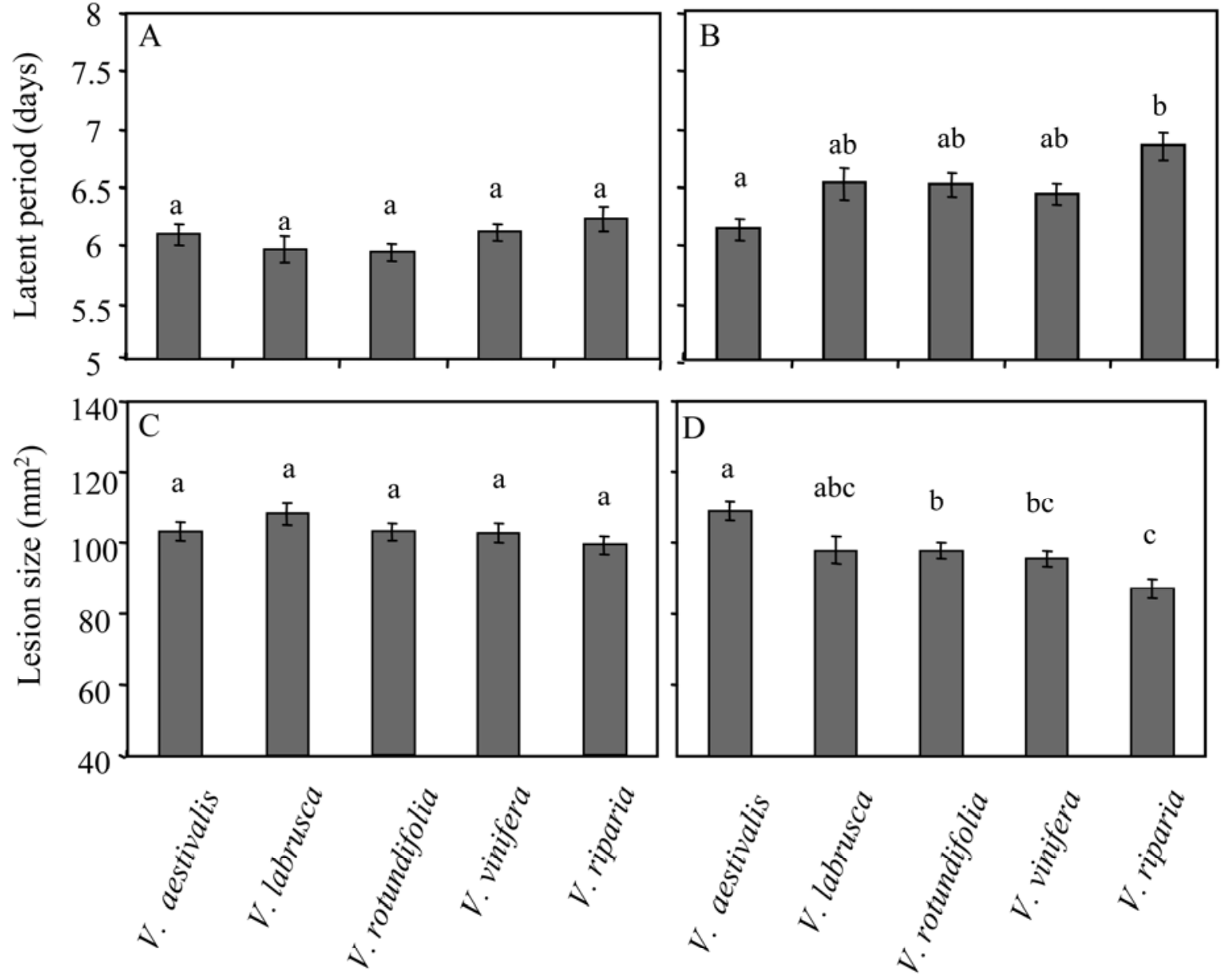

\section{Source hosts}

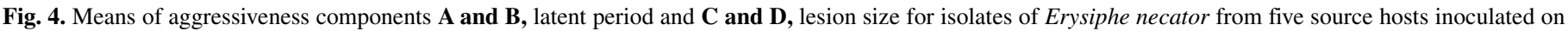

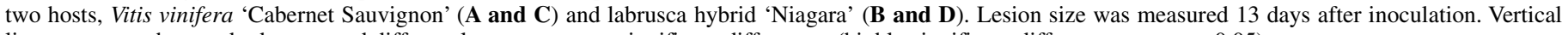
lines represent the standard errors and different letters represent significant differences (highly significant differences test, $\alpha=0.05$ ).

TABLE 4. Analysis of variance to determine the effect of geographic origin on the aggressiveness (latent period and lesion size) of 57 isolates of Erysiphe necator inoculated on two hosts, Vitis vinifera 'Cabernet Sauvignon' and labrusca hybrid 'Niagara'a

\begin{tabular}{|c|c|c|c|c|c|c|c|}
\hline \multirow[b]{2}{*}{ Source } & \multirow[b]{2}{*}{$\mathrm{df}$} & \multicolumn{3}{|c|}{ Latent period } & \multicolumn{3}{|c|}{ Lesion size } \\
\hline & & SS & $F$ ratio & Prob $>F$ & SS & $F$ ratio & Prob $>F$ \\
\hline Geographic region & 1 & 245,183 & 2.6635 & 0.1029 & 530,607 & 4.9170 & 0.0268 \\
\hline Inoculated host & 1 & $5,874,779$ & 63.8202 & $<0.0001$ & $1,059,327$ & 9.8164 & 0.0018 \\
\hline Experiment & 1 & 166,011 & 1.8034 & 0.1796 & $16,343,970$ & 151.4540 & $<0.0001$ \\
\hline
\end{tabular}

${ }^{a}$ Isolates were obtained from five Vitis spp. (source hosts) in two regions of the eastern United States: northeastern (Massachusetts, New Hampshire, Connecticut,

New York, New Jersey, Ohio, and Michigan; $n=24$ ) and southeastern (Georgia and North Carolina; $n=33$ ). 
indicates that it involves induction of programmed cell death specifically within the penetrated epidemic cell. Unfortunately, no information is available about resistance genes in the $V$. rotundifolia accession we inoculated in this study. Therefore, the $V$. rotundifolia-E. necator interaction might be a fertile ground for additional studies of host-pathogen interactions. Interestingly, we did not detect differences in aggressiveness (latent period and lesion size) between isolates from $V$. rotundifolia and those from V. vinifera or V. labrusca.

Pathogenicity of isolates from $V$. rotundifolia on all the Vitis hosts we tested and their equal aggressiveness as isolates from commonly cultivated source hosts imply that the population of $E$. necator on $V$. rotundifolia has the potential to be a source of inoculum for other Vitis spp. However, not a single isolate in our sample, including those from Vitis spp. in North Carolina (Hurdle Mills area) and Georgia (Ringgold area) sympatric with $V$. rotundifolia, was able to colonize $V$. rotundifolia, implying that gene flow from $V$. rotundifolia to other Vitis spp. is uncommon. This discrepancy has at least two possible explanations. First, the number of isolates we sampled from sympatric hosts was not large. By chance, we may not have sampled isolates from other Vitis spp. that could infect $V$. rotundifolia. Second, experimental inoculations in the laboratory (e.g., drop inoculations on detached leaves) do not always perfectly reflect pathogenesis in nature. For example, single-generation pathogenicity assays do not take into account other ecological factors which define fitness, such as differences related to overwintering survival, variation in sporulation and spore viability among isolates from different hosts (37), or epidemiological patterns on different host organs (34). Therefore, small differences in fitness among individuals measured in artificial conditions may translate into larger differences and niche partitioning in nature (34). To test these hypotheses, larger samples of isolates from sympatric hosts would need to be inoculated on $V$. rotundifolia. In addition, a complementary approach (also requiring larger samples) would be to genotype isolates with polymorphic genetic markers to test for genetic differentiation among Vitis spp. Estimating gene flow in field populations is likely to reveal more about the actual migration of E. necator under natural conditions, as opposed to the potential migration inferred from inoculations. This kind of population genetics approach would take into account multiple generations of selection acting on pathogen populations and avoid artifacts associated with inoculations done under artificial conditions (40).

In addition to complete host specialization on $V$. rotundifolia, we were able to detect significant variation in aggressiveness among isolates. We used the drop-inoculation technique described by Gadoury and Pearson (20) but we modified it for detached leaves, instead of in vitro plants and seedlings, which are harder to maintain and guard from contamination. Use of detached leaves also allowed us to assay a larger number of isolates than might be feasible with seedlings. Despite this modification, our results were consistent with those of Gadoury and Pearson (20) (i.e., we could detect significant differences among isolates on the resistant labrusca host, but not on the more susceptible host, $V$. vinifera). A major difference between our study and that of Gadoury and Pearson (20) is that we found significant differences in aggressiveness among isolates from different hosts and geographic origins. The most aggressive isolates were those from $V$. aestivalis, which produced larger lesions than isolates from the rest of the hosts (except for those from V. labrusca); their mean latent period was also significantly shorter than that of isolates from $V$. riparia. Although $V$. aestivalis is a common wild species in the eastern United States, most of the isolates we sampled were from the Appalachian Mountains, from relatively undisturbed and isolated environments. The distances between some sites where we sampled isolates from $V$. aestivalis in the southeast were more than 200 km (e.g., Blood Mt., GA and Marion, NC). In addition, isolates from $V$. aestivalis are not all clonal but belong to at least two different multilocus haplotypes (3). These findings exclude the possibility that we tested a single aggressive clone of $V$. aestivalis, which could have biased our results.

Few reports have compared the selective effect of quantitative resistance on pathogen aggressiveness. Gandon and Michalakis (22) hypothesized in their model that increased quantitative host resistance will select for higher pathogen aggressiveness. Cowger and Mundt (8) showed in field trials that individuals of Mycosphaerella graminicola with the highest level of aggressiveness were found on the more resistant cultivars. This phenomenon was also observed in Lettuce mosaic virus in lettuce (43). Although little is known about the mechanisms of quantitative resistance in wild Vitis spp., Fung et al. $(18,19)$ recently quantified the expression of defense-related genes in the hybrid cv. Norton (which they referred to as being $V$. aestivalis). Representative PR genes, including $P R-2$ and $P R-3$, were transcribed constitutively at higher levels and salicylic acid was found at higher levels in cv. Norton leaves than in $V$. vinifera. Additional expression profiling of different defense-related genes from labrusca hybrids, $V$. riparia, and $V$. rotundifolia and correlating them with aggressiveness measures of the pathogen populations might provide interesting findings on the possible connection between quantitative resistance in Vitis spp. and aggressiveness in E. necator.

Although $V$. vinifera is severely affected by powdery mildew wherever it is grown, individuals we sampled from this species were not significantly different in aggressiveness than those from other host species. This result may be related to the fact that $V$.
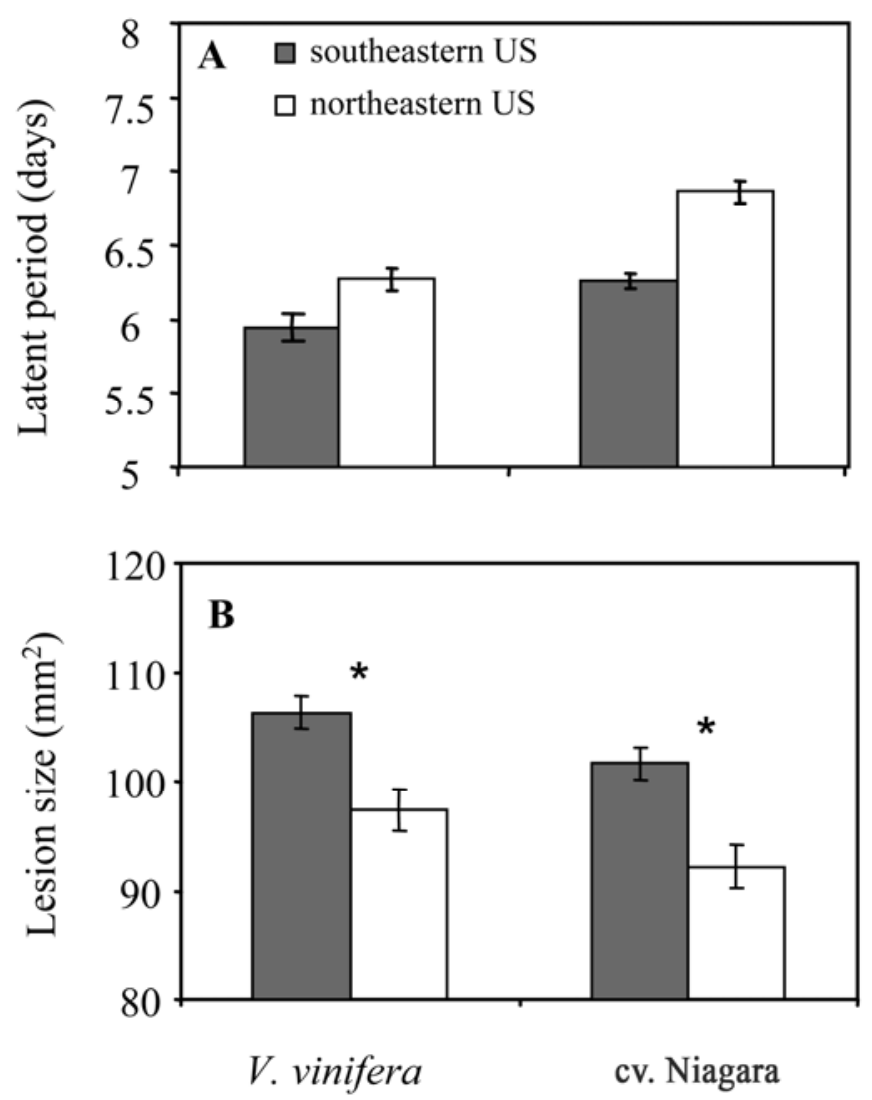

\section{Inoculated hosts}

Fig. 5. Mean aggressiveness components $\mathbf{A}$, latent period and $\mathbf{B}$, lesion size for isolates of Erysiphe necator from two geographic regions in the eastern United States: southeastern isolates ( $n=33$, solid bars) and northeastern isolates ( $n=24$, open bars) measured on two inoculated hosts: Vitis vinifera 'Cabernet Sauvignon' and labrusca hybrid 'Niagara'. Lesion size was measured 13 days after inoculation. Vertical lines represent the standard errors and asterisks indicate significant differences between regions $(P<0.01)$. 
vinifera is extremely susceptible to E. necator. The fact that no significant differences were detected between isolates from the different source hosts after inoculating them on $V$. vinifera supports the speculation that there is no significant selection on this host against individuals that were less aggressive on other Vitis spp. However, this hypothesis could be tested independently with genetic markers to assess the genetic structure of and estimate gene flow among populations of E. necator from $V$. vinifera compared with those from other Vitis hosts. Genetic markers and techniques for testing this type of hypothesis are currently being developed (unpublished results).

This research also found significant differences in aggressiveness between populations from different geographic regions because isolates from the southeast produced larger lesions than those from the northeast. This finding is consistent with inferences based on multilocus sequencing of restricted gene flow between populations of $E$. necator from commercial vineyards between these two regions (3). Diverse host-pathogen systems such as the plantain powdery mildew pathogen Podosphaera plantaginis and its host Plantago lanceolata (28), the yellow rust pathogen Puccinia striiformis f. sp. tritici and wheat in France, and the plant Lithophragma parviflorum and the floral-parasitic moth Greya politella in the northwestern United States (47) demonstrated that current co-evolutionary selection and interspecific interactions, including host-pathogen interactions, can be highly divergent across both narrow and wide geographic scales. Climatic factors differ markedly between the northeastern and southeastern United States. Many environmental factors such as summer and winter temperatures, wind, rain events, and UV radiation all potentially influence survival and dispersal of both sexual and asexual stages of E. necator $(9,50)$. In addition, the influences of these environmental factors on aggressiveness are well documented in other pathosystems. Thus, environmental influences on the ecology of E. necator may be fertile ground for further research. One issue which must be taken into consideration is the fact that the composition of Vitis spp., both wild and cultivated, differs between the northeast and the southeast. Except for $V$. labrusca and $V$. vinifera, sampling from other host species was unbalanced because $V$. rotundifolia does not grow in the northeast and $V$. riparia was uncommon in the sampling areas in the southeast. Therefore, we must be cautious when making conclusions about the differentiation of populations based on geography because it might be confounded by source hosts. On the other hand, the fact that isolates from $V$. aestivalis from the northeast were less aggressive than those from the southeast, the relatively short-distance dispersal of the pathogen by wind and rain (50), and the potential for selection by environmental factors is consistent with the potential for such differentiation by restricted gene flow. This possible source of host $\times$ geographic region interaction will surely need to be explored further.

The wild Vitis spp. in the eastern United States have been used for many years for pest and disease resistance breeding programs. However, complementary research on the genetics and biology of wild pathogens, and especially E. necator, are rare (20). We believe that a greater understanding of wild populations of $E$. necator would be beneficial for improving the collection of wild Vitis spp., screening for resistance, and contributing to the assessment of durability. E. necator is present in diverse geographic areas and landscapes and on wild Vitis hosts. In many places, we detected single plants late in the growing season with high severity of powdery mildew while adjacent plants of the same species were mildew-free. Despite the possibility that microclimatic parameters may explain part of these observations, we suggest that collecting germplasm under these conditions would allow a collector to distinguish between putatively resistant and susceptible individuals. Results of our study suggest the importance of including isolates from $V$. rotundifolia in screening for powdery mildew resistance trials of new muscadine cultivars and, more important, of new $V$. vinifera $\times V$. rotundifolia hybrids. Similarly, cultivars being genetically modified with transgenes from $V$. rotundifolia should also be screened with isolates of $E$. necator from $V$. rotundifolia to test whether they have the ability to overcome resistance.

We also showed variation among E. necator isolates for two quantitative components of aggressiveness. Because quantitative resistance is likely to play a prominent role in future breeding programs (14), it might be important to assess resistance against diverse E. necator populations, including challenging breeding material with $E$. necator isolates collected from wild species such as $V$. aestivalis, because they are more aggressive than those from other host species and may include diverse mechanisms with the potential to break the qualitative host resistance.

\section{ACKNOWLEDGMENTS}

This research was supported by Vaadia-BARD Postdoctoral Fellowship Award no. FI-410-2008 from the United States-Israel Binational Agricultural Research and Development Fund (BARD) to O. Frenkel, Research Travel Grant from the Cornell University Graduate School to M. T. Brewer, and Hatch project NYC-153410 to M. G. Milgroom. We thank all of the generous collaborators who helped us collect E. necator by assisting in our sampling at field sites and by sending powdery mildew samples, T. Sutton and S. Anas for helping us locate and collect samples in the field, P. Cousins for providing accessions of V. rotundifolia and V. aestivalis and for assistance in identifying Vitis spp., S. Sadigov and J. Booth for assistance with statistical analyses, and D. Gadoury and L. Cadle-Davidson for helpful advice throughout this project.

\section{LITERATURE CITED}

1. Ali, S., and Adhikari, T. B. 2008. Variation in aggressiveness of Stagonospora nodorum isolates in North Dakota. J. Phytopathol. 156:140145 .

2. Braun, U., and Takamatsu, S. 2000. Phylogeny of Erysiphe, Microsphaera, Uncinula (Erysipheae) and Cystotheca, Podosphaera, Sphaerotheca (Cystotheceae) inferred from rDNA ITS sequences-some taxonomic consequences. Schlechtendalia 4:1-33.

3. Brewer, M. T., and Milgroom, M. G. 2010. Phylogeography and population structure of the grape powdery mildew fungus Erysiphe necator from diverse Vitis species. BMC Evol. Biol. (In Press.)

4. Burdon, J. J., and Thrall, P. H. 2008. Pathogen evolution across the agroecological interface: implications for disease management. Evol. Appl. 1:57-65.

5. Chacón, M. G., Andrade-Piedra, J. L., Gessler, C., and Forbes, G. A. 2007. Aggressiveness of Phytophthora infestans and phenotypic analysis of resistance in wild Petota accessions in Ecuador. Plant Pathol. 56:549561.

6. Conover, W. J. 1998. Practical Nonparametric Statistics, 3rd ed. John Wiley \& Sons, New York.

7. Couch, B. C., Fudal, I., Lebrun, M. H., Tharreau, D., Valent, B., van Kim, P., Notteghem, J. L., and Kohn, L. M. 2005. Origins of host-specific populations of the blast pathogen Magnaporthe oryzae in crop domestication with subsequent expansion of pandemic clones on rice and weeds of rice. Genetics 170:613-630.

8. Cowger, C., and Mundt, C. C. 2002. Aggressiveness of Mycosphaerella graminicola isolates from susceptible and partially resistant wheat cultivars. Phytopathology 92:624-630.

9. Delp, J. C. 1954. Effect of temperature and humidity on the grape powdery mildew fungus. Phytopathology 44:651-626.

10. Délye, C., Laigret, F., and Corio-Costet, M. F. 1997. RAPD analysis provides insight into the biology and epidemiology of Uncinula necator. Phytopathology 87:670-677.

11. Donald, T. M., Pellerone, F., Adam-Blondon, A. F., Bouquet, A., Thomas, M. R., and Dry, I. B. 2002. Identification of resistance gene analogs linked to a powdery mildew resistance locus in grapevine. Theor. Appl. Genet. 104:610-618.

12. Doster, M. A., and Schnathorst, W. C. 1985. Comparative susceptibility of various grapevine cultivars to the powdery mildew fungus Uncinulanecator. Am. J. Enol. Vitic. 36:101-104.

13. Dry, I. B., Feechan, A., Anderson, C., Jermakow, A. M., Bouquet, A., Adam-Blondon, A. F., and Thomas, M. R. 2010. Molecular strategies to enhance the genetic resistance of grapevines to powdery mildew. Aust. J. Grape Wine Res. 16:94-105 
14. Eibach, R., Zyprian, E., Welter, L., and Topfer, R. 2007. The use of molecular markers for pyramiding resistance genes in grapevine breeding. Vitis 46:120-124.

15. Enjalbert, J., Duan, X., Leconte, M., Hovmølller, M. S., and De Vallavieille-Pope, C. 2005. Genetic evidence of local adaptation of wheat yellow rust (Puccinia striiformis f. sp. tritici) within France. Mol. Ecol. 14:2065-2073.

16. Evans, K. J., Whisson, D. L., and Scott, E. S. 1996. An experimental system for characterizing isolates of Uncinula necator. Mycol. Res. 100:675-680.

17. Frenkel, O., Peever, T. L., Chilvers, M. I., Özkilinc, H., Can, C., Abbo, S., Shtienberg, D., and Sherman, A. 2010. Ecological genetic divergence of the fungal pathogen Didymella rabiei on sympatric wild and domesticated Cicer spp. (chickpea). Appl. Environ. Microbiol. 76:30-39.

18. Fung, R. W. M., Gonzalo, M., Fekete, C., Kovacs, L. G., He, Y., Marsh, E., McIntyre, L. M., Schachtman, D. P., and Qiu, W. P. 2008. Powdery mildew induces defense-oriented reprogramming of the transcriptome in a susceptible but not in a resistant grapevine. Plant Physiol. 146:236-249.

19. Fung, R. W. M., Qiu, W. P., Su, Y. C., Schachtman, D. P., Huppert, K., Fekete, C., and Kovacs, L. G. 2007. Gene expression variation in grapevine species Vitis vinifera L. and Vitis aestivalis Michx. Genet. Res. Crop Evol. 54:1541-1553.

20. Gadoury, D. M., and Pearson, R. C. 1991. Heterothallism and pathogenic specialization in Uncinula necator. Phytopathology 81:1287-1293.

21. Galet, P. 1979. A Practical Ampelography: Grapevine Identification. Translated by L. T. Morton. Cornell University Press, Ithaca NY.

22. Gandon, S., and Michalakis, Y. 2000. Evolution of parasite virulence against qualitative or quantitative host resistance. Proc. R. Soc. B. 267:985-990.

23. Gee, C. T., Gadoury, D. M., and Cadle-Davidson, L. 2008. Ontogenic resistance to Uncinula necator varies by genotype and tissue type in a diverse collection of Vitis spp. Plant Dis. 92:1067-1073.

24. Giannakis, C., Bucheli, C. S., Skene, K. G. M., Robinson, S. P., and Scott, N. S. 1998. Chitinase and beta-1,3-glucanase in grapevine leaves: A possible defence against powdery mildew infection. Aust. J. Grape Wine Res. 4:14-22.

25. Gladieux, P., Zhang, X. G., Afoufa-Bastien, D., Valdebenito Sanhueza, R. M., Sbaghi, M., and Le Cam, B. 2008. On the origin and spread of the Scab disease of apple: out of central Asia. PLoS One 3:e1455.

26. Grünwald, N. J., and Flier, W. G. 2005. The biology of Phytophthora infestans at its center of origin. Annu. Rev. Phytopathol. 43:171-190.

27. Harlan, J. R. 1976. Diseases as a factor in plant evolution. Annu. Rev. Phytopathol. 14:31-51.

28. Laine, A. L. 2008. Temperature-mediated patterns of local adaptation in a natural plant-pathogen metapopulation. Ecol. Lett. 11:327-337.

29. Lenné, J. M., and Wood, D. 1991. Plant diseases and the use of wild germplasm. Annu. Rev. Phytopathol. 29:35-63.

30. Leppik, E. E. 1970. Gene centers of plants as sources of disease resistance. Annu. Rev. Phytopathol. 8:323-344.

31. Milgroom, M. G., and Fry, W. E. 1997. Contributions of population genetics to plant disease epidemiology and management. Adv. Bot. Res. 24:1-30.

32. Milus, E. A., Seyran, E., and McNew, R. 2006. Aggressiveness of Puccinia striiformis f. sp. tritici isolates in the south-central United States. Plant Dis. 90:847-852.

33. Montarry, J., Cartolaro, P., Delmotte, F., Jolivet, J., and Willocquet, L. 2008. Genetic structure and aggressiveness of Erysiphe necator popula- tions during grapevine powdery mildew epidemics. Appl. Environ. Microbiol. 74:6327-6332.

34. Montarry, J., Cartolaro, P., Richard-Cervera, S., and Delmotte, F. 2009. Spatio-temporal distribution of Erysiphe necator genetic groups and their relationship with disease levels in vineyards. Eur. J. Plant Pathol. 123:6170.

35. Olmo, H. P. 1971. Vinifera rotundifolia hybrids as wine grapes. Am. J. Enol. Vitic. 22:87-91.

36. Onokpise, O. U., and Mortensen, J. A. 1988. Genotype $\times$ environment interaction in Vitis rotundifolia Michaux. Am. J. Enol. Vitic. 39:121-124.

37. Pariaud, B., Ravigne, V., Halkett, F., Goyeau, H., Carlier, J., and Lannou, C. 2009. Aggressiveness and its role in the adaptation of plant pathogens. Plant Pathol. 58:409-424.

38. Pauquet, J., Bouquet, A., This, P., and Adam-Blondon, A. F. 2001. Establishment of a local map of AFLP markers around the powdery mildew resistance gene Run 1 in grapevine and assessment of their usefulness for marker assisted selection. Theor. Appl. Genet. 103:1201-1210.

39. Pearson, R. C., and Gadoury, D. M. 1992. Grape powdery mildew. In Plant Diseases of International Importance. Vol. III, Diseases of Fruit Crops. J. Kumar, H. S. Chaube, U. S., Singh, and A. N. Mukhopadhyay, eds. Prentice Hall, Englewood, NJ.

40. Peever, T. L., Olsen, L., Ibañez, A., and Timmer, L. W. 2000. Genetic differentiation and host specificity among populations of Alternaria spp. causing brown spot of grapefruit and tangerine $\times$ grapefruit hybrids in Florida. Phytopathology 90:407-414.

41. Péros, J. P., Nguyen, T. H., Troulet, C., Michel-Romiti, C., and Notteghem, J. L. 2006. Assessment of powdery mildew resistance of grape and Erysiphe necator pathogenicity using a laboratory assay. Vitis 45:29-36.

42. Péros, J. P., Troulet, C., Guerriero, M., Michel-Romiti, C., and Notteghem, J. L. 2005. Genetic variation and population structure of the grape powdery mildew fungus, Erysiphe necator, in southern France. Eur. J. Plant Pathol. 113:407-416.

43. Pink, D. A. C., Lot, H., and Johnson, R. 1992. Novel pathotypes of lettuce mosaic-virus - breakdown of a durable resistance. Euphytica 63:169-174.

44. Reisch, B. I., Goodman, R. N., Martens, M. H., and Weeden, N. F. 1993. The relationship between Norton and Cynthiana, red wine cultivars derived from Vitis aestivalis. Am. J. Enol. Vitic. 44:441-444.

45. Sacristán, S., and García-Arenal, F. 2008. The evolution of virulence and pathogenicity in plant pathogen populations. Mol. Plant Pathol. 9:369384.

46. Stukenbrock, E. H., and McDonald, B. A. 2008. The origins of plant pathogens in agro-ecosystems. Annu. Rev. Phytopathol. 46:75-100.

47. Thompson, J. N., and Cunningham, B. M. 2002. Geographic structure and dynamics of coevolutionary selection. Nature 417:735-738.

48. Thrall, P. H., Barrett, L. G., Burdon, J. J., and Alexander, H. M. 2005. Variation in pathogen aggressiveness within a metapopulation of the Cakile maritima-Alternaria brassicicola host-pathogen association. Plant Pathol. 54:265-274.

49. Wang, B., Brubaker, C. L., Tate, W., Woods, M. J., and Burdon, J. J. 2008. Evolution of virulence in Fusarium oxysporum f. sp. vasinfectum using serial passage assays through susceptible cotton. Phytopathology 98:296303.

50. Willocquet, L., Berud, F., Raoux, L., and Clerjeau, M. 1998. Effects of wind, relative humidity, leaf movement and colony age on dispersal of conidia of Uncinula necator, causal agent of grape powdery mildew. Plant Pathol. 47:234-242. 